\title{
Interferometers and Decoherence Matrices
}

\author{
D. Han* \\ National Aeronautics and Space Administration, Goddard Space Flight Center, Code 935, \\ Greenbelt, Maryland 20771 \\ Y. S. $\mathrm{Kim}^{\dagger}$ \\ Department of Physics, University of Maryland, College Park, Maryland 20742 \\ Marilyn E. Noz $\ddagger$ \\ Department of Radiology, New York University, New York, New York 10016
}

\begin{abstract}
It is shown that the Lorentz group is the natural language for two-beam interferometers if there are no decoherence effects. This aspect of the interferometer can be translated into six-parameter representations of the Lorentz group, as in the case of polarization optics where there are two orthogonal components of one light beam. It is shown that there are groups of transformations which leave the coherency or density matrix invariant, and this symmetry property is formulated within the framework of Wigner's little groups. An additional mathematical apparatus is needed for the transition from a pure state to an impure state. Decoherence matrices are constructed for this process, and their properties are studied in detail. Experimental tests of this symmetry property are possible.

42,25,Ja, 02.20.-a
\end{abstract}

Typeset using REVTEX

*electronic mail: han@trmm.gsfc.nasa.gov

†electronic mail: yskim@physics.umd.edu

‡electronic mail: noz@nucmed.med.nyu.edu 


\section{INTRODUCTION}

In our earlier papers [1, 2,, ,, 3$]$, we have formulated polarization optics in terms of the twoby-two and four-by-four representations of the six-parameter Lorentz group. It was noted that the two-component Jones vector and the four-component Stokes parameters are like the relativistic spinor and the Minkowskian four-vector respectively. We were able to identify the attenuator, rotator, and phase shifter with appropriate transformation matrices of the Lorentz group. It was noted that the two-element Jones vector is like the two-component Pauli spinor and that the four Stokes parameters act like the elements of a Minkowskian four-vector.

The purpose of this paper is to show that the mathematics of polarization optics is applicable also to interferometers. Our reasoning is that polarization optics is basically the physics of two plane waves. The same is true for two-beam interferometers. We need mathematical devices which will perform phase shifts between the waves and which will take care of attenuations at different rates. In the case of interferometers, it is possible to achieve the beam split and synthesis by rotation matrices. We can use the matrices of the above-mentioned Lorentz group in order to achieve these basic physical operations.

In addition, in this paper, we discuss the mathematical device which will describe the decoherence effect due to random phases. For this purpose, we need density matrices. However, the coherency matrix serves as the density matrix, and its four elements constitute the four components of the Stokes vector [5,6]. It was noted in our previous paper that it is possible to construct a four-by-four decoherence matrix which will transform a pure-state Stokes vector into a mixed-state Stokes vector. Unlike the case of attenuations, rotations, or beam splits and syntheses, the decoherence matrix does not belong to the Lorentz group.

In order to study the decoherence process more carefully, we borrow the concept of Wigner's little group originally developed for studying internal space-time symmetries of elementary particles [7,8]. Wigner's little group is the maximal subgroup of the Lorentz group whose transformations leave the four-momentum of a given particle invariant. In the present case, the little group consists of transformations on a given density matrix which will leave that matrix invariant. It is shown in this paper that the little group for pure states is like that for massless particles, while the little group for impure states is like that for massive particles. The transition of the little group from a pure to impure state is discussed in detail.

In Sec. II, we show how each element in the two-beam interferometer system corresponds to a transformation matrix in the Lorentz group. The combined effect is the two-by-two representation of the six-parameter Lorentz group. In Sec. III, it is pointed out that the coherency matrix can also be defined for the interferometer system and that this matrix serves as the density matrix. The transformation property of the density matrix is discussed in detail. In Sec. [V], we introduce the little group which will leave a given density matrix invariant. It is noted that the little group for pure states has a symmetry property quite different from that for impure states. In Sec. V, the decoherence matrices are discussed in detail. Although the augmentation of this matrix to the Lorentz group leads to a large group, there exist subgroups exhibiting symmetry properties familiar to us. Possible experiments with the decoherence matrix are suggested. 


\section{FORMULATION OF THE PROBLEM}

Typically, one beam is divided into two by a beam splitter. We can write the incoming beam as

$$
\Psi=\left(\begin{array}{l}
\psi_{1} \\
\psi_{2}
\end{array}\right)=\left(\begin{array}{c}
\exp \{i(k z-\omega t)\} \\
0
\end{array}\right)
$$

Then, the beam splitter can be written in the form of a rotation matrix [9]:

$$
R(\theta)=\left(\begin{array}{cc}
\cos (\theta / 2) & -\sin (\theta / 2) \\
\sin (\theta / 2) & \cos (\theta / 2)
\end{array}\right)
$$

which transforms the column vector of Eq.(1) into

$$
\left(\begin{array}{l}
\psi_{1} \\
\psi_{2}
\end{array}\right)=\left(\begin{array}{c}
{[\cos (\theta / 2)] \exp \{i(k z-\omega t)\}} \\
-[\sin (\theta / 2) \exp \{i(k z-\omega t)\}
\end{array}\right) .
$$

The first beam $\psi_{1}$ of Eq.(1) is now split into $\psi_{1}$ and $\psi_{2}$ of Eq.(3). The intensity is conserved. If the rotation angle $\theta$ is $-\pi / 4$, the initial beam is divided into two beams of the same intensity and the same phase [10].

These two beams go through two different optical path lengths, resulting in a phase difference. If the phase difference is $\phi$, the phase shift matrix is

$$
P(\phi)=\left(\begin{array}{cc}
e^{-i \phi / 2} & 0 \\
0 & e^{i \phi / 2}
\end{array}\right)
$$

When reflected from mirrors, or while going through beam splitters, there are intensity losses for both beams. The rate of loss is not the same for the beams. This results in the attenuation matrix of the form

$$
\left(\begin{array}{cc}
e^{-\eta_{1}} & 0 \\
0 & e^{-\eta_{2}}
\end{array}\right)=e^{-\left(\eta_{1}+\eta_{2}\right) / 2}\left(\begin{array}{cc}
e^{\eta / 2} & 0 \\
0 & e^{-\eta / 2}
\end{array}\right)
$$

with $\eta=\eta_{2}-\eta_{1}$. This attenuator matrix tells us that the electric fields are attenuated at two different rates. The exponential factor $e^{-\left(\eta_{1}+\eta_{2}\right) / 2}$ reduces both components at the same rate and does not affect the degree of polarization. The effect of polarization is solely determined by the squeeze matrix

$$
S(\eta)=\left(\begin{array}{cc}
e^{\eta / 2} & 0 \\
0 & e^{-\eta / 2}
\end{array}\right)
$$

In the detector or the beam synthesizer, the two beams undergo a superposition. This can be achieved by the rotation matrix like the one given in Eq.(2) [9]. For instance, if the angle $\theta$ is $90^{\circ}$, the rotation matrix takes the form

$$
\frac{1}{\sqrt{2}}\left(\begin{array}{cc}
1 & -1 \\
1 & 1
\end{array}\right)
$$

If this matrix is applied to the column vector of Eq.(3), the result is 


$$
\frac{1}{\sqrt{2}}\left(\begin{array}{c}
\psi_{1}-\psi_{2} \\
\psi_{1}+\psi_{2}
\end{array}\right)
$$

The upper and lower components show the interferences with negative and positive signs respectively.

We have shown in our previous papers that repeated applications of the rotation matrices of the form of Eq.(2), shift matrices of the form of Eq.(4) and squeeze matrices of the form of Eq.(6) lead to a two-by-two representation of the six-parameter Lorentz group. The transformation matrix in general takes the form

$$
G=\left(\begin{array}{ll}
\alpha & \beta \\
\gamma & \delta
\end{array}\right)
$$

applicable to the column vector of Eq.(1), where all four elements are complex numbers with the condition that the determinant of the matrix be one.

Although we can borrow all the elegant mathematical identities of the two-by-two representations of the Lorentz group, this formalism does not allow us to describe the loss of coherence within the interferometer system. In order to study this effect, we have to construct the coherency matrix:

$$
C=\left(\begin{array}{ll}
S_{11} & S_{12} \\
S_{21} & S_{22}
\end{array}\right)
$$

with

$$
\begin{array}{ll}
S_{11}=<\psi_{1}^{*} \psi_{1}>, & S_{22}=<\psi_{2}^{*} \psi_{2}>, \\
S_{12}=<\psi_{1}^{*} \psi_{2}>, & S_{21}=<\psi_{2}^{*} \psi_{1}>.
\end{array}
$$

It is sometimes more convenient to use the following combinations of parameters.

$$
\begin{aligned}
& S_{0}=S_{11}+S_{22}, \\
& S_{1}=S_{11}-S_{22}, \\
& S_{2}=S_{12}+S_{21}, \\
& S_{3}=-i\left(S_{12}-S_{21}\right) .
\end{aligned}
$$

These four parameters are called the Stokes parameters in the literature [11, 12], usually in connection with polarized light waves. In the present paper, we are applying these parameters to two separate beams in a given interferometer system.

The Stokes parameters, originally developed for polarization optics, are becoming applicable to other branches of physics dealing with two orthogonal states. In this paper, we are using these parameters for interferometers.

We have shown previously [2] that the four-by-four transformation matrices applicable to the Stokes parameters are like Lorentz-transformation matrices applicable to the spacetime Minkowskian vector $(t, z, x, y)$. This allows us to study space-time symmetries in terms of the Stokes parameters which are applicable to interferometers. Let us first see how the rotation matrix of Eq.(2) is translated into the four-by-four formalism. In this case,

$$
\alpha=\delta=\cos (\theta / 2), \quad \gamma=-\beta=\sin (\theta / 2) .
$$


The corresponding four-by-four matrix takes the form [4]

$$
R(\theta)=\left(\begin{array}{cccc}
1 & 0 & 0 & 0 \\
0 & \cos \theta & -\sin \theta & 0 \\
0 & \sin \theta & \cos \theta & 0 \\
0 & 0 & 0 & 1
\end{array}\right)
$$

Let us next see how the phase-shift matrix of Eq.(田) is translated into this fourdimensional space. For this two-by-two matrix,

$$
\alpha=e^{-i \phi / 2}, \quad \beta=\gamma=0, \quad \delta=e^{i \phi / 2} .
$$

For these values, the four-by-four transformation matrix takes the form [4]

$$
P(\phi)=\left(\begin{array}{cccc}
1 & 0 & 0 & 0 \\
0 & 1 & 0 & 0 \\
0 & 0 & \cos \phi & -\sin \phi \\
0 & 0 & \sin \phi & \cos \phi
\end{array}\right)
$$

For the squeeze matrix of Eq.(6),

$$
\alpha=e^{\eta / 2}, \quad \beta=\gamma=0, \quad \delta=e^{-\eta / 2} .
$$

As a consequence, its four-by-four equivalent is

$$
S(\eta)=\left(\begin{array}{cccc}
\cosh \eta & \sinh \eta & 0 & 0 \\
\sinh \eta & \cosh \eta & 0 & 0 \\
0 & 0 & 1 & 0 \\
0 & 0 & 0 & 1
\end{array}\right)
$$

If the above matrices are applied to the four-dimensional Minkowskian space of $(t, z, x, y)$, the above squeeze matrix will perform a Lorentz boost along the $z$ or $S_{1}$ axis with $S_{0}$ as the time variable. The rotation matrix of Eq.(14) will perform a rotation around the $y$ or $S_{3}$ axis, while the phase shifter of Eq.(16) performs a rotation around the $z$ or the $S_{1}$ axis. Matrix multiplications with $R(\theta)$ and $P(\phi)$ lead to the three-parameter group of rotation matrices applicable to the three-dimensional space of $\left(S_{1}, S_{2}, S_{3}\right)$.

The phase shifter $P(\phi)$ of Eq.(16) commutes with the squeeze matrix of Eq.(18), but the rotation matrix $R(\theta)$ does not. This aspect of matrix algebra leads to many interesting

mathematical identifies which can be tested in laboratories. One of the interesting cases is that we can produce a rotation by performing three squeezes [ $[$ ]. Another interesting case is a combination of squeeze and rotation will produce a matrix which will convert numerical multiplication into addition. This aspect known as the Iwasawa decomposition is discussed in detail in Ref. [四].

\section{DENSITY MATRICES AND THEIR LITTLE GROUPS}

According to the definition of the density matrix [6], the coherency matrix of Eq.(10) is also the density matrix. Since we discussed transformation properties of coherency matrices in our earlier papers [1,2], we can start here with those results on this subject. 
The most effective way of formulating the symmetry property of a given physical system is to construct a group of transformations which leave the system invariant. This concept was originally developed by Wigner [7] for internal space-time symmetries of relativistic particles. Wigner's little group is the maximal subgroup of the Lorentz group whose transformations leave the four-momentum of a given particle invariant. For instance, for a particle at rest, the little group is the three-parameter rotation group. The rotations do not change the four-momentum of the particle, even though they change the direction of the spin. There are also massless particles which cannot be brought to rest. This is the reason why the little group for a massive particle is different from that of the massless particle. The little group for massless particles is like (or locally isomorphic to) the two-dimensional Euclidean group [7,8].

Indeed, in Ref. [4, we discussed Wigner rotations and Iwasawa decompositions rotations applicable to massive and massless particles respectively and how these little-group transformations can be applied to the Stokes four-vectors. In this section, we shall see that the Stokes vectors for pure and impure states are like the four-momentum of the massless and massive particles respectively.

In the following discussion, we will need transformations of the Stokes four-vectors and the corresponding transformations of the two-by-two density matrices. We are quite familiar with four-by-four matrices applicable to the Stokes vectors. For the two-by-two density matrices, the transformation takes the form

Under the influence of the $G$ transformation given in Eq.(9), this coherency matrix is transformed as

$$
\begin{aligned}
C^{\prime}= & G C G^{\dagger}=\left(\begin{array}{ll}
S_{11}^{\prime} & S_{12}^{\prime} \\
S_{21}^{\prime} & S_{22}^{\prime}
\end{array}\right) \\
& =\left(\begin{array}{ll}
\alpha & \beta \\
\gamma & \delta
\end{array}\right)\left(\begin{array}{ll}
S_{11} & S_{12} \\
S_{21} & S_{22}
\end{array}\right)\left(\begin{array}{ll}
\alpha^{*} & \gamma^{*} \\
\beta^{*} & \delta^{*}
\end{array}\right),
\end{aligned}
$$

where $C$ and $G$ are the density matrix and the transformation matrix given in Eq.(10) and Eq.(9) respectively. According to the basic property of the Lorentz group, these transformations do not change the determinant of the density matrix $C$. Transformations which do not change the determinant are called unimodular transformations.

As we shall see in this section, the determinant for pure states is zero, while for that for mixed states does not vanish. Is there then a transformation matrix which will change this determinant within the Lorentz group. The answer is No. This is the basic issue we would like to address in this section.

If the phase difference between the two waves remains intact, the the system is said to in a pure state, and the density matrix can be brought to the form

$$
\left(\begin{array}{ll}
1 & 0 \\
0 & 0
\end{array}\right),
$$

through the transformation of Eq.(19) with a suitable choice of the $G$ matrix. For the pure state, the Stokes four-vector takes the form

$$
\left(\begin{array}{l}
1 \\
1 \\
0 \\
0
\end{array}\right)
$$


In order to study the symmetry properties of the density matrix, let us ask the following question. Is there a group of transformation matrices which will leave the above density matrix invariant? In answering this question, it is more convenient to use the Stokes fourvector. The column vector of Eq.(21) is invariant under the operation of the phase shifter $P(\phi)$ of Eq.(16). In addition, it is invariant under the following two matrices:

$$
\begin{aligned}
& F_{1}(u)=\left(\begin{array}{cccc}
1+u^{2} / 2 & -u^{2} / 2 & u & 0 \\
u^{2} / 2 & 1-u^{2} / 2 & u & 0 \\
u & -u & 1 & 0 \\
0 & 0 & 0 & 1
\end{array}\right), \\
& F_{2}(v)=\left(\begin{array}{cccc}
1+v^{2} / 2 & -v^{2} / 2 & 0 & v \\
v^{2} / 2 & 1-v^{2} / 2 & 0 & v \\
0 & 0 & 1 & 0 \\
u & -v & 0 & 1
\end{array}\right) .
\end{aligned}
$$

These mathematical expressions were first discovered by Wigner in 1939 [7] in connection with the internal space-time symmetries of relativistic particles. They went through a stormy history, but it is gratifying to note that they serve a useful purpose for studying interferometers where each matrix corresponds to an operation which can be performed in laboratories.

The $F_{1}$ and $F_{2}$ matrices commute with each other, and the multiplication of these leads to the form

$$
F_{2}(u) F_{2}(v)=\left(\begin{array}{cccc}
1+\left(u^{2}+v^{2}\right) / 2 & -\left(u^{2}+v^{2}\right) / 2 & u & u \\
\left(u^{2}+v^{2}\right) / 2 & 1-\left(u^{2}+v^{2}\right) / 2 & u & v \\
u & -u & 1 & 0 \\
v & -v & 0 & 1
\end{array}\right)
$$

This matrix contains two parameters.

Let us go back to the phase-shift matrix of Eq.(16). This matrix also leaves the Stokes vector of Eq.(21) invariant. If we define the "little group" as the maximal subgroup of the Lorentz group which leaves a Stokes vector invariant, the little group for the Stokes vector of Eq.(21) consists of the transformation matrices given in Eq.(16) and Eq.(23).

Next, if the phase relation is completely random, and the first and second components have the same amplitude, the density matrix becomes

$$
\left(\begin{array}{cc}
1 / 2 & 0 \\
0 & 1 / 2
\end{array}\right)
$$

Here is the question: Is there a two-by-two matrix which will transform the pure-state density matrix of Eq.(20) into the impure-state matrix of Eq.(24)? The answer within the system of matrices of the form given in Eq.(9) is No, because the determinant of the purestate density matrix is zero while that for the impure-state matrix is $1 / 4$. Is there a way to deal with this problem? We shall return to this problem in Sec. $\nabla$. In this section, we restrict ourselves to the unimodular transformation of Eq.(19) which preserves the value of the determinant of the density matrix. The Stokes four-vector corresponding to the above density matrix is 


$$
\left(\begin{array}{l}
1 \\
0 \\
0 \\
0
\end{array}\right)
$$

This vector is invariant under both the rotation matrix of Eq.(14) and the phase shift matrix of Eq.(16). Repeated applications of these matrices lead to a three-parameter group of rotations applicable to the three-dimensional space of $\left(S_{1}, S_{2}, S_{3}\right)$.

Not all the impure-state density matrices take the form of Eq.(24). In general, if they are brought to a diagonal form, the matrix takes the form

$$
\frac{1}{2}\left(\begin{array}{cc}
1+\cos \chi & 0 \\
0 & 1-\cos \chi
\end{array}\right)
$$

and the corresponding Stokes four-vector is

$$
e^{-\eta}\left(\begin{array}{c}
\cosh \eta \\
\sinh \eta \\
0 \\
0
\end{array}\right)
$$

with

$$
\eta=\frac{1}{2} \ln \frac{1+\cos \chi}{1-\cos \chi}
$$

The matrix which transforms Eq.(25) to Eq.(27) is the squeeze matrix of Eq.(18). The question then is whether it is possible to transform the pure state of Eq.(21) to the impure state of Eq.(27) or to Eq.(25).

In order to see the problem in terms of the two-by-two density matrix, let us go back to the pure-state density matrix of Eq.(20). Under the rotation of Eq.(2),

$$
\begin{aligned}
& \left(\begin{array}{cc}
\cos (\chi / 2) & -\sin (\chi / 2) \\
\sin (\chi / 2) & \cos (\chi / 2)
\end{array}\right)\left(\begin{array}{ll}
1 & 0 \\
0 & 0
\end{array}\right) \\
& \times\left(\begin{array}{cc}
\cos (\chi / 2) & \sin (\chi / 2) \\
-\sin (\chi / 2) & \cos (\chi / 2)
\end{array}\right),
\end{aligned}
$$

the pure-state density matrix becomes

$$
\frac{1}{2}\left(\begin{array}{cc}
1+\cos \chi & \sin \chi \\
\sin \chi & 1-\cos \chi
\end{array}\right)
$$

For the present case of two-by-two density matrices, the trace of the matrix is one for both pure and impure cases. The trace of the $(\text { matrix })^{2}$ is one for the pure state, while it is less than one for impure states.

The next question is whether there is a two-by-two matrix which will eliminate the offdiagonal elements of the above expression that will also lead to the expression of Eq.(26). In order to answer this question, let us note that the determinant of the density matrix vanishes for the pure state, while it is non-zero for impure states. The Lorentz-like transformations 
of Eq.(19) leave the determinant invariant. Thus, it is not possible to transform a pure state into an impure state by means of the transformations from the six-parameter Lorentz group. Then is it possible to achieve this purpose using two-by-two matrices not belonging to this group. We do not know the answer to this question. We are thus forced to resort to four-by-four matrices applicable to the Stokes four-vector.

\section{DECOHERENCE EFFECTS ON THE LITTLE GROUPS}

We are interested in a transformation which will change the density matrix of Eq.(20) to Eq.(24). For this purpose, we can use the Stokes four-vector consisting of the four elements of the density matrix. The question then is whether it is possible to find a transformation matrix which will transform the pure-state four-vector of Eq.(21) to the impure-state fourvector of Eq.(25).

Mathematically, it is more convenient to ask whether the inverse of this process is possible: whether it is possible to transform the four-vector of Eq.(25) to that of Eq.(21). This is known in mathematics as the contraction of the three-dimensional rotation group into the two-dimensional Euclidean group [8]. Let us apply the squeeze matrix of Eq.(18) to the four-vector of Eq.(25). This can be written as

$$
\left(\begin{array}{cccc}
\cosh \eta & \sinh \eta & 0 & 0 \\
\sinh \eta & \cosh \eta & 0 & 0 \\
0 & 0 & 1 & 0 \\
0 & 0 & 0 & 1
\end{array}\right)\left(\begin{array}{l}
1 \\
0 \\
0 \\
0
\end{array}\right)=\left(\begin{array}{c}
\cosh \eta \\
\sinh \eta \\
0 \\
0
\end{array}\right)
$$

After an appropriate normalization, the right-hand side of the above equation becomes like the pure-state vector of Eq.(21) in the limit of large $\eta$, as $\cosh \eta$ becomes equal to $\sinh \eta$ in the infinite- $\eta$ limit. This transformation is from a mixed state to a pure or almost-pure state. Since we are interested in the transformation from the pure state of Eq. (21) to the impure state of Eq.(25), we have to consider an inverse of the above equation:

$$
\left(\begin{array}{cccc}
\cosh \eta & -\sinh \eta & 0 & 0 \\
-\sinh \eta & \cosh \eta & 0 & 0 \\
0 & 0 & 1 & 0 \\
0 & 0 & 0 & 1
\end{array}\right)\left(\begin{array}{c}
\cosh \eta \\
\sinh \eta \\
0 \\
0
\end{array}\right)=\left(\begin{array}{l}
1 \\
0 \\
0 \\
0
\end{array}\right)
$$

However, the above equation does not start with the pure-state four-vector. If we apply the same matrix to the pure state matrix, the result is

$$
\left(\begin{array}{cccc}
\cosh \eta & -\sinh \eta & 0 & 0 \\
-\sinh \eta & \cosh \eta & 0 & 0 \\
0 & 0 & 1 & 0 \\
0 & 0 & 0 & 1
\end{array}\right)\left(\begin{array}{l}
1 \\
1 \\
0 \\
0
\end{array}\right)=e^{-\eta}\left(\begin{array}{l}
1 \\
1 \\
0 \\
0
\end{array}\right) .
$$

The resulting four-vector is proportional to the pure-state four-vector and is definitely not an impure-state four-vector.

The inverse of the transformation of Eq.(31) is not capable of bringing the pure-state vector into an impure-state vector. Let us go back to Eq.(31), it is possible to bring a 
impure-state into a pure state only in the limit of infinite $\eta$. Otherwise, it is not possible. It is definitely not possible if we take into account experimental considerations.

The story is different for the little groups. Let us start with the rotation matrix of Eq.(14), and apply to this matrix the transformation matrix of Eq.(31). Then

$$
\left(\begin{array}{cccc}
\cosh \eta & \sinh \eta & 0 & 0 \\
\sinh \eta & \cosh \eta & 0 & 0 \\
0 & 0 & 1 & 0 \\
0 & 0 & 0 & 1
\end{array}\right)\left(\begin{array}{cccc}
1 & 0 & 0 & 0 \\
0 & \cos \theta & -\sin \theta & 0 \\
0 & \sin \theta & \cos \theta & 0 \\
0 & 0 & 0 & 1
\end{array}\right)\left(\begin{array}{cccc}
\cosh \eta & -\sinh \eta & 0 & 0 \\
-\sinh \eta & \cosh \eta & 0 & 0 \\
0 & 0 & 1 & 0 \\
0 & 0 & 0 & 1
\end{array}\right)
$$

If $\eta$ is zero, the above expression becomes the rotation matrix of Eq.(14). If $\eta$ becomes infinite, it becomes the little-group matrix $F_{1}(u)$ of Eq.(22) applicable to the pure state of Eq.(21). The details of this calculation for the case of Lorentz transformations are given in the 1986 paper by Han et al. [13]. We are then led to the question of whether one little-group transformation matrix can be transformed from the other.

If we carry out the matrix algebra of Eq.(34), the result is

$$
\left(\begin{array}{cccc}
1+\alpha u^{2} w / 2 & -\alpha u^{2} w / 2 & \alpha u w & 0 \\
\alpha u^{2} w / 2 & 1-u^{2} w / 2 & u w & 0 \\
\alpha u w & -u w & 1-\left(1-\alpha^{2}\right) u^{2} w / 2 & 0 \\
0 & 0 & 0 & 1
\end{array}\right)
$$

where

$$
\alpha=\tanh \eta, \quad u=-2 \tan \left(\frac{\theta}{2}\right), \quad w=\frac{1}{1+\left(1-\alpha^{2}\right) \tan ^{2}(\theta / 2)} .
$$

If $\alpha=0$, the above expression becomes the rotation matrix of Eq.(14). If $\alpha=1$, it becomes the $F_{1}$ matrix of Eq.(22). Here we used the parameter $\alpha$ instead of $\eta$. In terms of this parameter, it is possible to make an analytic continuation from the pure state with $\alpha=1$ to an impure state with $\alpha<1$ including $\alpha=0$.

On the other hand, we should keep in mind that the determinant of the density matrix is zero for the pure state, while it is non-zero for all impure states. For $\alpha=1$, the determinant vanishes, but it is nonzero and stays the same for all non-zero values of $\alpha$ less than one and greater than or equal to zero. The analytic expression of Eq.(36) hides this singular nature of the little group [13].

\section{DECOHERENCE MATRICES}

We are interested in the decoherence effect on the density matrix. We are particularly interested in the mechanism where the off-diagonal elements $S_{12}$ and $S_{21}$ become smaller due to time average or phase-randomizing process [14]. If this happens, we can apply to the Stokes four-vector the following decoherence matrix.

$$
\left(\begin{array}{cccc}
1 & 0 & 0 & 0 \\
0 & 1 & 0 & 0 \\
0 & 0 & e^{-2 \lambda} & 0 \\
0 & 0 & 0 & e^{-2 \lambda}
\end{array}\right)
$$


which can also be written as

$$
e^{-\lambda}\left(\begin{array}{cccc}
e^{\lambda} & 0 & 0 & 0 \\
0 & e^{\lambda} & 0 & 0 \\
0 & 0 & e^{-\lambda} & 0 \\
0 & 0 & 0 & e^{-\lambda}
\end{array}\right)
$$

where $e^{-\lambda}$ is the overall decoherence factor. For convenience, we define the decoherence matrix as

$$
D(\lambda)=\left(\begin{array}{cccc}
e^{\lambda} & 0 & 0 & 0 \\
0 & e^{\lambda} & 0 & 0 \\
0 & 0 & e^{-\lambda} & 0 \\
0 & 0 & 0 & e^{-\lambda}
\end{array}\right)
$$

This matrix cannot be constructed from the six-parameter Lorentz group applicable to the Stokes four-vectors.

If we combine this decoherence matrix with the Lorentz group, the result will be a fifteenparameter group of four-by-four matrices isomorphic to $O(3,3)$ which is beyond the scope of the present paper [15]. In order to extract the symmetry of physical interest, let us go back to the four-by-four matrices $R(\theta), P(\phi)$, and $S(\eta)$ of Eq.(14), Eq.(16), and Eq.(18) respectively. The phase-shift matrix of Eq.(16) commutes with the decoherence matrix.

As we discussed in our earlier paper on polarization optics [2], the decoherence matrix and the rotation matrix will lead to two-dimensional squeeze transformations applicable to the two-component vector

$$
V_{A}=\left(\begin{array}{c}
S_{1} \\
S_{2}
\end{array}\right)
$$

The four-by-four $D(\lambda)$ matrix of Eq.(39) and the rotation matrix of Eq.(14) become reduced to

$$
\begin{aligned}
D_{A}(\lambda) & =\left(\begin{array}{cc}
e^{\lambda} & 0 \\
0 & e^{-\lambda}
\end{array}\right), \\
R_{A}(\theta) & =\left(\begin{array}{cc}
\cos \theta & -\sin \theta \\
\sin \theta & \cos \theta
\end{array}\right) .
\end{aligned}
$$

As for the remaining components of the Stokes parameters, we can define another twocomponent vector as

$$
V_{B}=\left(\begin{array}{c}
S_{0} \\
S_{3}
\end{array}\right)
$$

The decoherence matrix applicable to this two-component vector is

$$
D_{B}(\lambda)=\left(\begin{array}{cc}
e^{\lambda} & 0 \\
0 & e^{-\lambda}
\end{array}\right)
$$

but the rotation matrix does not change the two-component vector $V_{B}$. 
Let us go back to the two-dimensional space of $V_{A}$, and its two-by-two transformation matrices. The matrices $D_{A}(\lambda)$ and $R_{A}(\theta)$ of Eq.(41) applicable are strikingly similar to the two-by-two matrices given in Eq.(6) and Eq.(2) respectively. If we replace the parameters $\eta$ in $S(\eta)$ and $\theta$ in $R(\theta)$ by $2 \lambda$ and $2 \theta$ respectively, they become $D_{A}$ and $R_{B}$ of Eq.(41).

With these two matrices, we can repeat the calculations for the Wigner rotations and Iwasawa decompostions discussed in our earlier paper [4]. It is possible to perform experiments to test these mathematical relations.

\section{CONCLUDING REMARKS}

In this paper, we have discussed two-beam interferometers within the framework of the six-parameter Lorentz group. It has been shown that beam splitters and beam synthesizers can be represented by two-by-two rotation matrices. The phase shift can also be represented by two-by-two rotation matrices applicable to spinor systems. As for attenuation, we introduced two-by-two squeeze matrices. The combined effect of these transformations leads to a two-by-two representation of the six-parameter Lorentz group.

We have found that the mathematical formalism given in this paper is identical to the formalism we presented in our earlier papers for polarization optics. In this series of papers, our purpose has been to minimize the group theoretical language and write down formulas close to what we observe in the real world. In this paper, we were able to by-pass completely the group theoretical formality known as the Lie algebra of the Lorentz group consisting of generators and their closed commutation relations.

With this improved mathematical technique, we discussed two-beam physics in terms of the little groups using only matrices which are realizable in laboratories. It has been shown that the little groups for pure and impure states are different. It was noted that analytic continuation from a pure state to an impure state is possible for the little groups. On the other hand, this transformation does not exist within the six-parameter Lorentz group, but requires an extra four-by-four matrix applicable to the Stokes four-vector, called the decoherence matrix.

The augmentation of this decoherence matrix into the Lorentz group will lead to a bigger group which is beyond the scope of this paper [15]. However, this bigger group has $O(2,1)$ like or $S U(1,1)$-like subgroups which are quite familiar to us from the squeezed states of light, and the Lorentz group-formulation of the polarization optics [2]. We are fortunate to observe, within the framework of this decoherence matrix, mathematical consequence which will lead to experiments on Wigner rotations and Iwasawa decompositions which are possible in both polarization optics and interferometers.

It will be a challenging problem to translate what we did in this paper to the language of quantum optics. The rotation operations corresponding to phase shifts and rotations around the direction of the propagation can be formulated in terms of the two-mode squeezed states [16]. However, the squeeze transformations discussed in this paper correspond to the loss of intensity, which cannot be translated into quantum optics. On the other hand, the decoherence matrix can be accommodated into the density-matrix formalism. Indeed, they all are challenging problems.

Furthermore, unlike the case of polarization optics, there can be more than two beams for interferometers. For instance, three-beam interferometer are quite common. This will 
open up a new research line for studying symmetry properties in optics. The power of group theoretical approaches is that we can establish the symmetry properties in one branches of physics to those in a different field using the isomorphism and/or homomorphism of group theory. As for the three-beam case, we are happy to note a recent paper by Rowe et al. 18. 


\section{REFERENCES}

[1] D. Han, Y. S. Kim, and M. E. Noz, J. Opt. Soc. Am. A 14, 2290 (1997).

[2] D. Han, Y. S. Kim, and M. E. Noz, Phys. Rev. E 56, 6065 (1997);

[3] For earlier and later papers on this subject, see R. Barakat, J. Opt. Soc. Am. 53(3) 317 (1963); C. S. Brown and A. E. Bak, Opt. Engineering 34, 1625 (1995); J. J. Monzon and L. L. Sánchez-Soto, Phys. Lett. A 26218 (1999).

[4] D. Han, Y. S. Kim, and M. E. Noz, Phys. Rev. E 60, 1036 (1999).

[5] M. Born and E. Wolf, Principles of Optics, 6th Ed. (Pergamon, Oxford, 1980). The first edition of this book was published in 1959 .

[6] R. P. Feynman, Statistical Mechanics (Benjamin/Cummings, Reading, MA, 1972).

[7] E. Wigner, Ann. Math. 40, 149 (1939).

[8] Y. S. Kim and M. E. Noz, Theory and Applications of the Poincaré Group (Reidel, Dordrecht, 1986); Y. S. Kim and M. E. Noz, Phase Space Picture of Quantum Mechanics (World Scientific, Singapore, 1991).

[9] B. C. Sanders and A. Mann, Group 22, Proceedings of the 22nd International Colloquium on Group Theoretical Methods in Physics, S. P. Cornel et al. eds. (International Press, Boston, 1999). See pp 474-478.

[10] For earlier papers on beam splitters based on the $S U(2)$ and $S p(2)$ transformations, see R. A. Campos, B. E. A. Saleh, and M. C. Teich, Phys. Rev. A, 40, 1371 (1989) and A. Luis and L. L. Sánchez-Soto, Quantum Semniclass. Opt. 7, 153 (1995), respectively.

[11] W. A. Shurcliff, Polarized Light (Harvard Univ. Press, Cambridge, MA, 1962).

[12] E. Hecht, Am. J. Phys. 38, 1156 (1970).

[13] D. Han, Y. S. Kim, and D. Son, J. Math. Phys. 27, 2228 (1986).

[14] D. F. McAlister and M. G. Raymer, Phys. Rev. A 55, R1609 (1997).

[15] D. Han, Y. S. Kim, and M. E. Noz, J. Math. Phys. 36, 3940 (1995).

[16] B. Yurke, S. McCall, and J. R. Klauder, Phys. Rev. A 33, 4033 (1986).

[17] D. Han, Y. S. Kim, and D. Son, 1987, Class. Quantum Grav. 4, 1777 (1987).

[18] D. J. Rowe, B. C. Sanders, and H. de Guise, J. Math. Phys. 40, 3604 (1999). 